\title{
Hyperspectral Image Classification Using CNN12
}

\author{
Mr. Ruhikesh Dhande ${ }^{*}$, Rohit Miri² and S.R.Tandan ${ }^{3}$ \\ ${ }^{1}$ Department of Information Technology, Sipna COET. Amravati, India \\ ${ }^{2}$ Department of Computer Science, Dr. C. V. Raman University, Chhattisgarh, India \\ ${ }^{3}$ Department of Computer Science, Dr. C. V. Raman University, Chhattisgarh, India
}

\section{ABSTRACT}

Convolutional neural networks (CNNs) have showed their dominance in hyperspectral image classification (HIS) where spectral embedded with spatial features have provided means for excellent database training when the application is image classification. Different scaling with context of number of layers in efficient neural networks has been the recent topic of research where attention aided and not aided machine learning algorithm have being the elementary topic of vigilance. We have in the paper highlighted an attention seeking CNN 12 layer algorithm that is utilized for efficient labeling the prime aspect of image classification. The raw image provided as input to the network extracts the relevant features in a way that joining feature map created provides better results in terms of accuracy. The frame work created utilizes the available sets of images and compares the parameters that will help in making the algorithm robust and fine tuned with respect to required hyper parameters. The linear transformation that is the main advantage of CNN architecture has made the system more reliable unlike the rule based feature extraction and classification frameworks. In this paper the three dimensional input utilization helps us solve the overfitting problem that is a byproduct of exhaustive layering during training. The way the three dimensional input is handled in terms of CNN 12 layer convoluting is the simple nonlinear function that is the key aspect of this research paper to improve accuracy.

KEY WORDS: IMAGE CLASSIFICATION, HIS, CNN.

\section{INTRODUCTION}

Hyperspectral remote sensing via Hyperspectral imagery (HSI) is the requirement of providing multispectral processing capabilities where normal RGB imaging provides insufficient capabilities. This requirement of handling multiple spectral information not only requires a strong back bone of processing speed but no compensation in terms of accuracy can be subdued where minimizing the feature extracted is concerned. HIS has been evolving from the past decade as the hindrances of handling large

\section{ARTICLE INFORMATION}

${ }^{*}$ Corresponding Author: rdhande92@gmail.com

Received 17th Oct 2020 Accepted after revision 29th Dec 2020

Print ISSN: 0974-6455 Online ISSN: 2321-4007 CODEN: BBRCBA

Thomson Reuters ISI Web of Science Clarivate Analytics USA and Crossref Indexed Journal

\section{1) Clarivate $\begin{gathered}\text { Analytics } \\ \text { rossef }\end{gathered}$}

NAAS Journal Score 2020 (4.31)

A Society of Science and Nature Publication,

Bhopal India 2020. All rights reserved.

Online Contents Available at: http//www.bbrc.in/

Doi: $h t t p: / / d x$.doi.org/10.21786/bbrc/13.14/106 datasets has been evolving constantly where competition always has been with respect to providing an efficient machine learning algorithm. This algorithm was not only meant to be custom made for the problems of classification but the problem needed to be addressed is also evolving with the needs of making remote sensing solve problems especially in the area of agriculture. The early ear of algorithm and framework development was restricted to spectral classifiers where the machine is to be supervised or non supervised depended totally on the application coverage.

Linear and non linear regression provided a good start but soon multilingual utilization brought demands to extract physical properties hence brought about classifiers like support vector machines (SVM), random forest(RF) etc. These classifiers did the job but custom tailoring these classifiers increased the over fitting problems that required handling capabilities of an efficient trained data base. Recent reporting's have showcased that the 
combination of methods provided better approaches to deal with the spectral as well as spatial data. These methods adaptation was with respect to utilization of advantages of one classification like spectral information and the other classification with respect to spatial. These adjoining feature mapping was possible with respect to various profiles that could be integrated with spatial based methods reported like the joint sparse model etc. The reporting quishuo et all. have utilized a novel two parallel pathway unlike a singular means of CNN structure that is the main motivation of utilizing CNN in our research.

One more immediate recent reported work RadhesyamVaddiet all has utilized a data normalization process to down scale the loss parameters. This not only reduced the computational burden but also provided the required integration facility that $\mathrm{CNN}$ architecture promised. This paper is based on Probabilistic Principal Component Analysis (PPCA), Gabor filtering and Convolutional Neural Network(CNN) which is batch normalization approach to improve accuracy. In this work, PPCA, gabor filtering and CNN provided scalar factoring to be merged with batch normalization and dropout mechanism that increased accuracy. The framework is essential and provides excellent breakthrough where in the structuring is restricted by the researcher to three sets of convolution and pooling layers each. This layering and filtering when fed to batch normalization brings about the lower limits on batch size hence the author has restricted his use to 3 layering each. With the goal of reducing computation time and providing further accuracy in this research paper we have utilized and provided a layer normalization function framework instead of batch normalization which peaks out to provide a balance between layering and algorithm in terms of simplicity and accuracy. The paper is organized as Section II is methodology, Section III is implementation and Section IV results and discussion.

\section{METHODOLOGY}

The working procedure comprises of HIS classification wherefirst the input acquired HIS data is normalized for the spatial features extracted. The normalization is layer based where in the multidimension input for our case the 3D data corresponds to the feature data in terms of height width and depth in general terms. One of the key difference of layer normalization unlike batch normalization is that it does not require for formulation of running mean and variance. It can be mathematical expressed as shown in the below equations.

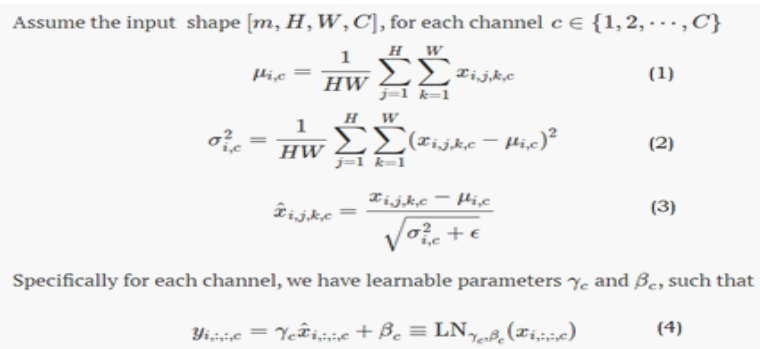

As each sample to train is an neuron that requires combustive instantiation the researcher utilized multiple channels to be normalized as shown in the figure at pooling. The spectral features are convoluted before fusion with the scale of neuron filtering that is varies with respect to $2 \mathrm{~N}$ where $\mathrm{n}$ is the number of layers from 1 to 12 and filters are required at each layer to control the number of feature maps created for labeling in a convolution neural network. For fusion pooling operation is performed which is responsible to study the non linear properties that are divide at each layer in terms of dimensionality. This pooling is done at each layer so that most relevant information is extracted as down sampling of data is provided on each stride configured.

On general basis the data remains constant at each layer. This is very essential for our algorithm to overcome overfitting configurations at the very base itself. The pooling that we utilize is the average pooling which is required for processing the normalized data Next step is variation in convolution which a measure technique where in the function utilized is bounded. This bounded variation defined as BV function which is determined by real values under bounding extents. Bounded convolution is varied in terms of the axis definition rows and columns that in our case is $\mathrm{y}$ axis where $\mathrm{x}$ axis is kept constant or neglected for keeping the values finite to hyperplane (hypersurface or hyper parameter tuning). In terms of mathematical equation the bounded variation two measures $\mu$ and $\nu$ based convolution can be written as shown below.

$$
\begin{aligned}
& \int_{\mathbf{R}^{d}} f(x) d(\mu * \nu)(x)=\int_{\mathbf{R}^{d}} \int_{\mathbf{R}^{d}} f(x+y) d \mu(x) d \nu(y) . \\
& \text { In particular, } \\
& (\mu * \nu)(A)=\int_{\mathbf{R}^{d} \times \mathbf{R}^{d}} 1_{A}(x+y) d(\mu \times \nu)(x, y),
\end{aligned}
$$

III. Implementation: For the described methodology the desired framework is developed as shown in figure 1 and analyzed on the four standard hyper spectral data sets(Indian Pines, Salinas, Pavia University and Pavia).

Figure 1: Block Diagram of implemented CNN framework

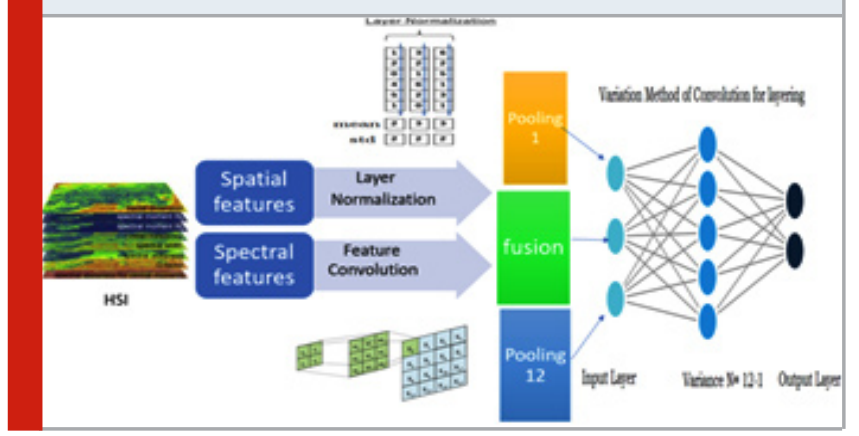

The complete description of the data sets summarized is highlighted in table 1. The experimentation is done considering the spatial resolution size mainly so that the effect of increase in size can be observed on accuracy. 
The implementation can be noted down into step of operation as shown below and diagrammatically with respect to figure 2 . The python code is developed on the principles of figure 2 .

1. HSI classification framework for spatial and spectral feature extraction.

2. Data normalization - with respect to layer.

3. Spatial and Spectral data fusion and pooling.

4. Bounding Variance Convolution.

5. CNN 12 Layer based feature labeling.

Table 1. Data set description

\begin{tabular}{|c|c|c|c|c|}
\hline $\begin{array}{c}\text { Sr } \\
\text { NO }\end{array}$ & $\begin{array}{c}\text { Name of } \\
\text { the } \\
\text { Dataset }\end{array}$ & $\begin{array}{c}\text { Number } \\
\text { of } \\
\text { Classes }\end{array}$ & $\begin{array}{c}\text { Spatial } \\
\text { Resolution }\end{array}$ & $\begin{array}{c}\text { No of } \\
\text { bands }\end{array}$ \\
\hline 1 & Indian Pines & 16 & $145 \times 145$ & 224 \\
\hline 2 & Salinas & 16 & $610 \times 340$ & 200 \\
\hline 3 & Pavia University & 9 & $610 \times 610$ & 103 \\
\hline 4 & Pavia Centre & 9 & $1096 \times 1096$ & 102 \\
\hline
\end{tabular}

Figure 2: CNN 12 feature Extraction

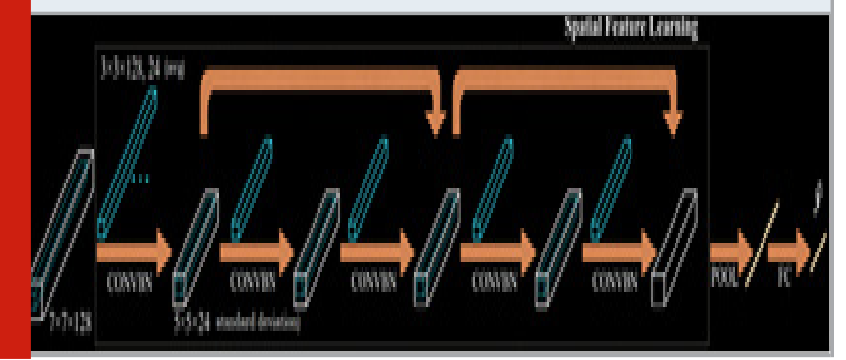

\section{RESULTS AND DISCUSSION}

A demonstration screenshot of result with respect to python output is shown in figure 3. The results of all the databases with respect to group accuracy and loss function is summarized and compared as shown in table 2 .

Figure 3 : Implemented output stored in text files.

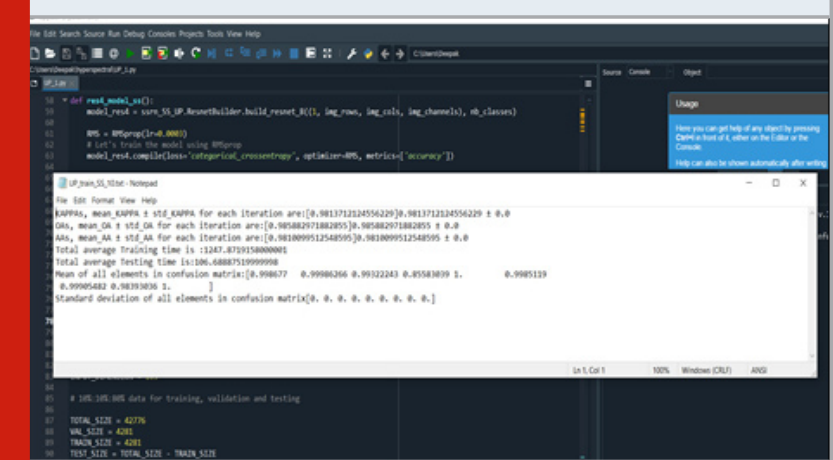

The output of the proposed model is plotted with the help of matplotlib and the corresponding figures of each data base analyzed is shown in terms of Figure 4.a Ground truth, Figure 4.b Classification Maps, Figure 4.c layer coverage with respect to classification and figure 4.d shows the linear correlation output. The main advantage of using the layer normalization is that the network provides better accuracy in terms of bounding power. The amount of testing time is also improved to approximately 100 seconds as shown in figure 3 . The kappa coefficient are the hyper parameter tuning that have improved the loss function as compared to reported literature. The hyper parameter tuning with respect to individual class sub function requires exhaustive testing which is sub due to machine/server capability. Hence exact comparison of the reported literature is the salient setback that brings the user ambiguity in terms of software and machine utilization in true sense. This fine tuning and removal of ambiguity is the future scope of our research.

Table 2. Accuracy and loss calculations

\begin{tabular}{|l|c|c|c|c|}
\hline \multirow{2}{*}{$\begin{array}{l}\text { Name of } \\
\text { the Dataset }\end{array}$} & \multicolumn{2}{|c|}{ Reference algorithm } & \multicolumn{2}{c|}{ CNN 12* } \\
\cline { 2 - 5 } & Accuracy & Loss & Accuracy & Loss \\
\hline Indian Pines & 0.9902 & 0.0355 & 0.9964 & 0.041 \\
\hline Salinas & 0.9994 & 0.0033 & 0.9956 & 0.006 \\
\hline Pavia University & 0.9994 & 0.0032 & 0.9941 & 0.008 \\
\hline Pavia Centre & $\begin{array}{c}\text { Not } \\
\text { Analyzed }\end{array}$ & $\begin{array}{c}\text { Not } \\
\text { Analyzed }\end{array}$ & 0.9928 & 0.009 \\
\hline
\end{tabular}

Figure 4: (a) Ground truth, (b) Classification Maps, (c) Layer Coverage, (d) Linear correlation output.

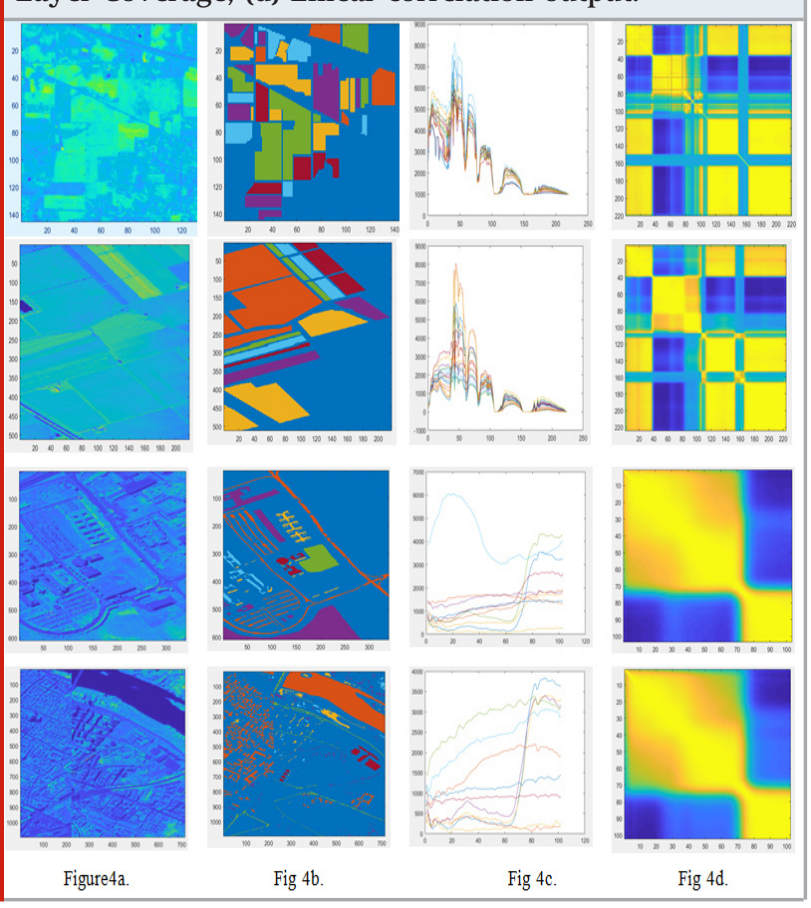




\section{REFERENCES}

DANIEL GOELEVEN, "Variation method with application to convolution equations", journal of integral equations and analysis, Volume 6, number 3, 1994.

Gabriel Zaid, Lilian Bossuet, AmauryHabrard, Alexandre venelli, “ Methodology for efficient CNN architectures in profiling attacks”, Research gate, July 2019, DOI: 10.13154/tches.v2020.i1.1-36

Hyperspectral Remote Sensing Scenes - http://www.ehu. eus/ccwintco/index.php?title=Hyperspectral_Remote_ Sensing_Scenes

QishuoGao,Samsung Lim, andXiuping Jia, “
Hyperspectral Image Classification Using Convolutional Neural Networks and Multiple Feature Learning”, MDPI, Remote Sens. 2018, https://doi.org/10.3390/ rs 10020299

RadhesyamVaddi, PrabukumarManoharan, "Hyperspectral image classification using CNN with spectral and spatial features integration", Infrared Physics \& Technology, Volume 107, June 2020, 103296

Rushiksh Dhande,Dr. Rohit Miri, Dr. S. Tandan, Database Training: Requirement to Exploitation in Hyperspectral Imagery. 